\title{
Cessation strategies used successfully by individuals in recovery from methamphetamine addiction
}

Yvonne Gordon and Christine Stephens, School of Psychology, Massey University, New Zealand

\begin{abstract}
INTRODUCTION: Methamphetamine (MA) misuse is a recognised health issue in Aotearoa New Zealand, and there is a lack of appropriate treatment available for individuals who are methamphetamine dependent. This exploratory study, undertaken in 2019, sought to gain insight from individuals in Aotearoa who have experienced MA dependence and now identify as being in recovery, to discover which strategies, approaches or treatment appeared helpful in their recovery.
\end{abstract}

METHODS: The participants in the study were seven adults (New Zealand European, Samoan and Māori ethnicity) who had abstained from methamphetamine for six months or more. In-depth interviews were audiotaped and transcribed before being analysed. The data were analysed using interpretative phenomenological analysis, which has its theoretical origins in phenomenology and hermeneutics.

FINDINGS: Four themes emerged to describe the lived experience of recovery from methamphetamine misuse: Getting Away, Support, Personal Sources of Strength, and Treatment. Each theme held importance in the participants' recovery from MA and provided insight into their journey in abstaining and being in recovery.

CONCLUSIONS: These findings may be used to assist others entering recovery. The present findings are limited by the size of the sample; however, they provide valuable information on this important health issue as a basis for further research, which is urgently needed in Aotearoa.

KEYWORDS: Methamphetamine; health issue; recovery; treatment

AOTEAROA

NEW ZEALAND SOCIAL WORK 33(2), 32-44.

CORRESPONDENCE TO: Yvonne Gordon

evegordon88@gmail.com
MA has been recognised as one of the most frequently used drugs worldwide, and an important health issue that requires close attention (European Monitoring Centre for Drugs and Drug Addiction, 2018). It is a synthetic drug which is a member of a family of drugs known as central nervous system stimulants, titled amphetamine-type stimulants, comprised of amphetaminesgroup substances (Weisheit \& William, 2009). MA comes as a clear, crystal-like compound or as a powder that can be used by snorting, injecting, inhaling or ingesting (Fields, 2017). Individuals use MA for reasons which can include physical or mental performance enhancement, experimentation as a social activity, escapism from inner issues, or through peer pressure. There are aspects of an individual's environment, biology or own personality and genetic makeup that can cause them to be more susceptible to the drug. This can include mental illness, socioeconomic status, social relationships, culture/ethnicity, hereditary predisposition, 
and trauma-related events (New Zealand Drug Foundation, 2018).

The issue of MA in Aotearoa New Zealand, as in other countries, has steadily escalated since the late 1990s (UNODC, 2004). The 2017/ 18 New Zealand Health Survey results for amphetamine use in the previous 12 months indicate a prevalence of $0.7 \%$, which is an estimated 26,000 adults. This is a lower percentage than $2015 / 16$. The survey found that participants aged between $24-34$ (1.5\%) had the highest prevalence, followed by 15-24 (1\%), while ages 55-64 (0.1\%) had the lowest, which shows the same pattern as in past surveys. Māori use (2.3\%) remains comparatively different from non-Māori use $(0.7 \%)$. The most recent government statistics show that the overall numbers of people who use MA in Aotearoa New Zealand has remained relatively consistent in the past five years (New Zealand Drug Foundation, 2018).

MA is a powerfully addictive stimulant that poses a great risk to overall wellbeing. MA has a short half-life (the time it takes for half of the drug to no longer be in the bloodstream) and therefore the user will often repeat use, which results in the onset of rapid dependence in comparison with other drugs such as alcohol. The cross-over from the initial time an individual uses MA to when they reach loss of control and become dependent on the drug can be rapid for MA users, and generally, dependence on the drug occurs and then controls the individual's behaviour long before they acknowledge it or can see what has happened (Halkitis, 2009).

Heavy use can result in neglect of self-care routines such as sleeping, eating, personal hygiene and exercise, which can lead to sleep deprivation, malnutrition, and dental damage. Long-term use can cause anxiety, depression, and damage to the nervous system and susceptibility to infectious disease. The lack of sleep can induce drug psychosis, and intravenous use increases the risk of contracting diseases such as HIV / AIDS (Gordon \& De Jong, 2018). Users can have occasional episodes of sudden and violent behaviour, intense paranoia, visual and auditory hallucinations. There can also be a tendency to compulsively clean and groom, and repetitively sort and disassemble objects such as cars and other mechanical devices, or to spend hours invested in a hobby that involves the user's own handywork and concentration (Halkitis, 2009). The effects have a devastating impact on an MA user's life as they often neglect their families, friends, and communities. They then often lose their jobs, money, and family and can hit "absolute rock bottom" before trying to do something about the crippling effects this drug has had on their lives (Roll et al., 2009).

Despite their desire to use less, in a review of treatment research, Halkitis (2009) states that MA users do not typically seek help until they present with severe consequences. This is typically because they enjoy the drug's effects too much to want to seek support, and when they do seek help as the damaging effects become clear, their prolonged use often means they are by then highly addicted to the drug (Jenner \& Lee, 2008). The literature suggests there is a range of individual and service level barriers that can prevent MA users from getting treatment. Barriers can include embarrassment or stigma, lack of funding and resources, privacy concerns, lack of awareness regarding treatment options, limited access to services, and lack of supports (Halkitis, 2009). Another issue undermining the success of MA treatment options is the significant shortage of providers with expertise in this specific drug addiction and an unavailability of appropriate services (Cannon, 2018).

The withdrawal symptoms that are experienced by MA users, even after long periods of abuse, do not include physical withdrawal symptoms like vomiting and shaking. MA use creates more of a psychological dependence than a physical 
dependence and therefore withdrawal is more likely to produce psychological symptoms such as depression, anxiety, and cravings (Drug Abuse, 2019). However, both dependence and addiction are treatable conditions. The goal is to separate the individual from the drug in a slow and controlled way to allow the body to readjust to a normal level of functioning (Drug Abuse, 2019). To date, no single modality to treat MA dependence has been identified as fully effective in communitybased, clinical or research settings, so this is already a barrier for individuals to be integrated into an appropriate and successful treatment. However, there are some treatment approaches with some evidence of efficacy, with further knowledge required to understand what else may be helpful. At present, the Matrix Model remains one of the most publicised and well-respected approaches to addressing MA addiction (Cannon, 2018). Cognitive Behavioural Therapy and Contingency Management are also well supported counselling-based treatment approaches, and studies show that the contingency management approach can be useful in conjunction with the behavioural therapy as it allows the individual to engage in other negotiated or contracted desirable behaviours (Weisheit \& William, 2009). Research shows that overall outcomes for MA users are improved by establishing a firm relationship (rapport), family support for the user, greater treatment satisfaction, and longer retention in treatment (McKetin et al., 2018).

Information collected by the New Zealand Ministry of Health has indicated a general increase in the number of individuals attending mental health and addiction services with a diagnosis of abuse or dependence on MA. Between 2011 and 2012, the number of individuals with a primary diagnosis of Amphetamine-Type Stimulants (ATS) abuse or dependence doubled, and significant numbers of those identified as having ATS use as a secondary issue when attending mental health and addiction services (New Zealand Ministry of Health, 2013). The pattern of contact with services in more recent years is also reflected in data provided by the Alcohol Drug Helpline, which reports that, in 2013, they were receiving approximately 300 calls about MA use every few months. These calls were mainly coming from family concerned about someone else's MA use, which is consistent with the pattern of MA users not seeking help until they present with severe consequences (Alcohol Drug Helpline, 2019).

Depending on the length and severity of dependence on MA, some individuals may be able to successfully manage their own withdrawal symptoms in the community without external support, or with support from a GP, whereas some individuals will have the need for greater support when going through withdrawal. In Aotearoa New Zealand there are Community Alcohol and Drug Services and other agencies which offer counselling, family therapy, detox programmes in a hospital or at home, medication, and referral to residential treatment programmes if required (Meth Xpert NZ, 2019). Despite there being services in Aotearoa New Zealand to cater for individuals who suffer from drug and alcohol issues, the problem faced by individuals with dependence on MA is the lack of services available or providers who cater specifically for treating MA (New Zealand Drug Foundation, 2018).

Most research on methamphetamine misuse, treatment and recovery has been conducted overseas rather than within Aotearoa New Zealand, so utilising the knowledge discovered overseas has been essential in developing our own framework and guidelines. In 2009, the government made an action plan to address the rising issue of MA in Aotearoa New Zealand, and, as a direct result of this, the New Zealand Ministry of Health instigated that Matua Raki (the national centre for addiction workforce development in Aotearoa New Zealand) make use of the overseas research and write guidelines for treatment providers. In 2010, 
Matua Raki released the comprehensive report "Interventions and Treatment for Problematic Use of Methamphetamine and Amphetamine-Type Stimulants (ATS)." In 2013, Matua Raki released a further review in a follow-up to the earlier report (New Zealand Ministry of Health, 2010, 2013).

The reports were prepared to review what treatment for MA works and what does not, and to review current and emerging developments in the treatment of problematic MA use. They were intended to be a working guide for those working with individuals affected by MA including frontline workers, Mental Health Services, police, general practitioners, addiction treatment providers, counsellors, and emergency departments. They support and recommend the few treatment modalities that have been recommended from overseas studies including the Matrix Model, Contingency Model, and working with high dose users in rehabilitation treatment centres (New Zealand Ministry of Health, 2010). There is support for these reviews from educational providers for MA such as the Dual Recovery Network in Christchurch who promote these reviews to help improve treatment in Aotearoa New Zealand and are advocating to support further use of the Matrix Model into the New Zealand health system (Dual Recovery Network, 2016). However, at present, there is still a need for an expansion in both the number of treatment services that are provided and awareness for the population group on what is available to them (New Zealand Drug Foundation, 2018).

The lack of available services has led to frustration in communities, which has led to grassroots type approaches in attempts at reducing MA use. There are drop-in centres in some regions of New Zealand run voluntarily by ex-addicts and supporters under a group called "P Pull," treatment facilities which are stretched to capacity with long waiting lists, and there are a few rehabilitation centres which offer specialised services for MA users that have waiting lists and eligibility criteria for potential clients (New Zealand Herald, 2019). There is also a rehabilitation centre in the Hutt Valley, near Wellington, which is called "Red Door Recovery." Red Door offers the "Pipe Down Meth Recovery Programme" which is specifically designed to meet the unique and difficult challenges presented by methamphetamine (Red Door Recovery, 2018). In Auckland there is a rehabilitation centre called "Higher Ground" which has an eight-bed contract through the Prime Minister's Methamphetamine Strategy, as well as accepting methamphetamine clients into other funded beds in the wider programme. Findings from the Higher Ground 2011-2014 outcome review indicate that Higher Ground is effective in working with MA clients, with $94 \%$ abstinent at 3 months when followed up at 3, 6, 9, and 12 months post-discharge, and $87 \%$ abstinent at 12 months (King, 2014). Te Whare Oranga Ngakau is a residential alcohol and drug treatment centre in Rotorua which focuses on providing clinical services within a Kaupapa Māori framework. The 24-hour residential programme has 15 AOD beds and two methamphetamine beds and offers a 12-week programme, or longer if required (Māori and Addiction Treatment Services, 2012).

A study was carried out in Aotearoa New Zealand in 2010 to discover the most commonly used interventions with MA from six agencies who dealt with the treatment of MA. The most common interventions were discovered to be Te Whare Tapa Whā and CBT, followed by harm reduction, motivational interviewing relapse prevention, behaviour therapy, brief therapy, group- and person-centred therapy (Cobzaru, 2010). Although the Matrix Model and Contingency Model were not mentioned, the wide use of Te Whare Tapa Whā is a therapeutic approach that is culturally appropriate for Māori, and consistent with Māori values and cultural identity (New Zealand Ministry of Health, 2010).

When looking at drug dependence and recovery, it is important to pay particular 
attention to which population group has the highest prevalence of use to target interventions for this population group and ensure there are appropriate services and treatment available. In the most recent $\mathrm{New}$ Zealand Health Survey, the highest rates of use were in males, people aged from 24-35 years, and people of Māori ethnicity. There was a study carried out in Aotearoa New Zealand in 2010 to discover the most commonly used interventions with MA from six agencies who dealt with the treatment of MA. The most common interventions were found to be Te Whare Tapa Whā and CBT, followed by harm reduction, motivational interviewing relapse prevention, behaviour therapy, brief therapy, group- and personcentred therapy (Cobzaru, 2010). Although the Matrix Model and Contingency Model were not mentioned, it is pleasing to see the wide use of Te Whare Tapa Whā, which is a therapeutic approach that is culturally appropriate for Māori, and consistent with Mãori values and cultural identity (New Zealand Ministry of Health, 2010).

Providing effective treatment for MA dependence is a growing need. There is knowledge that can be readily sourced on what the drug is, its effects, how it is manufactured, the seizure rates and trafficking of the drug, but less is known about the treatment options and how well these are working. It has been established that there is a need to look further at treatment options and recovery (Stoneberg et al., 2017). The study was aimed at examining the experiences of those identifying as being in recovery from MA dependence; at gaining insight into what approaches have helped or contributed to their journey of recovery, from the individual's perspective; and at prompting further research on the area of treatment and recovery.

\section{Methods}

\section{Methodology}

This study was motivated by the first author noticing the harm MA was causing for individuals and wider communities in Aotearoa New Zealand through coverage in the media as well as insights gained from facilitating mental health treatment for her clients. Despite this concern, the first author was committed when interviewing the participants, to have utmost respect, objectivity and nonjudgement when gaining insight into their personal experiences.

To gain an understanding of the lived experience of MA recovery, Miller et al. (2010) suggest that qualitative research is important in capturing how substance use and addiction are lived, and uncovering what the experience of recovery is like for individuals. Hermeneutic phenomenology was the theoretical framework for this research.

\section{Participants}

The criteria for participant selection was that the participants were over the age of 18 years and had abstained from MA for a period of six months or more. The participants needed to be both willing and able to articulate their lived experience of identifying as "in recovery from MA misuse" and the strategies, treatment or approaches that they have found helpful in allowing them to achieve this.

Snowball recruitment was the method employed to recruit participants. This involved finding a participant, interviewing them and then asking if they knew others who may be potential participants for the research and asking them to provide those potential participants with some information on the research, and researcher contact details (Flick, 2014).

Each individual who expressed interest in being a participant was checked to ensure they met the criteria and either emailed or posted a detailed information sheet, which outlined what the research was about, what they would be asked to do, what the information would be used for, and what they could expect from the researcher. This 
was a successful recruitment method and resulted in seven participants.

Semi-structured interviews were conducted with the participants in 2019. The participants were four males and three females. Ethnicities included European, Samoan, and Māori. Their ages at the time of interviewing ranged from 32 years to 53 years, with a median age of 36 years.

\section{Interviews}

The interview length was approximately one hour per participant. The interviews were conducted by the first author who used the same interview guide for each interview. The format was semi-structured, and focus was placed on allowing the participant to tell their story and describe their experiences. On completion of each interview the researcher explained what would happen with the information, which included the data being transcribed and analysed in order to form research findings, and how it would be stored.

The participants were asked if they would like a copy of the transcript to ensure they were happy with the data and make any edits they saw necessary. None of the participants chose to have a copy of the transcript and all participants consented to having their interviews audio-taped; tapes were then transcribed and analysed. The participants were provided a "summary of findings" on completion of the study.

Ethical considerations addressed included consent, data collection, documentation, recording, sharing research findings, benefits / risks, participant recruitment, privacy / confidentiality, Treaty of Waitangi and cultural considerations. The procedures were approved by the Massey University Human Ethics Committee before the start of recruitment and data collection.

\section{Data analysis}

Interpretative phenomenological analysis (IPA) was used because of its idiographic focus on phenomena that relate to experiences of some personal significance to individuals, such as drug dependence (Savin-Baden \& Major, 2013). IPA is intended for a small number of participants, so was ideal for this study with seven participants. Once each interview had taken place, the researcher immediately began transcribing the interview before reading and scrutinising the data to cluster the data into common themes to develop the descriptions of experience (Moustakas, 1994).

The first author used a data analysis software program to code the data. Once the data were coded and themes were found, the emergent themes were listed on a separate document so that the researcher could look for cross-participant themes. There were 10-12 themes identified. Some of the common themes were clustered together, which reduced the findings to four main themes (Smith, 2008).

\section{Results}

The participants in this research had used MA for a period of between six months and 17 years, with a median time of six years. Participants talked about their experiences of MA use and the consequences this had on their lives. They described how they stopped caring about other people and that their life revolved around the drug. The majority talked about being in unstable and unhealthy relationships throughout their time of using. They became estranged from their friends and family and socially withdrew from them due to the ongoing dependence they had on MA. Participants talked about the various physical health problems they had experienced: becoming very thin from not eating, barely sleeping, pimples/scabs on their body, trouble with their teeth, and one participant had permanent lockjaw which he believed was from MA use.

Participants said that they struggled with terrible "comedowns" from MA and the majority experienced psychotic episodes or paranoia from the prolonged use of MA. 
For some participants, the paranoia and psychotic episodes resulted in violence, aggression and getting involved in criminal activity and trouble with the law. Some participants also suffered from mental health problems while they were using MA and were trying to manage this alongside their MA use. Participants also spoke about seeing the first-hand consequences that MA had for other users such as health damage, immorality, illegal behaviour, and suicide. These observations and insights were enough to encourage them to analyse their own lives and assess that, perhaps, that was not the lifestyle they wanted to lead or the type of future they saw for themselves. This motivated them to enter their recovery journey.

Four common themes emerged as being significant to the participants' recovery from MA. These were labelled: Support, Getting Away, Treatment and Personal Sources of Strength. The first theme, Support, describes the importance that support from others had in their recovery journey. The second, Getting Away, describes how participants sought to stay away from other users and dealers, moving location, and cutting ties. The third theme, Treatment, incorporates the participants' inner issues, treatment, and education to better understand how these have impacted on their recovery. The last theme, Personal Sources of Strength, discusses individual factors that participants expressed as being helpful to their recovery. Quotations from participants used as examples of the data are presented below.

\section{Support}

All participants identified support as being crucial to their recovery. Support was identified as coming from family, partners, and treatment services. For some of the participants, their first step into recovery was surrendering and admitting to family that they needed help with their MA use, after realising that they no longer had control over it and were experiencing negative consequences. This was an important step because, while using MA, many of the participants had distanced themselves from their own families and friends. This also meant that participants felt obliged to tell the truth, which had not always been the case when they were trying to hide their drug use. When they did seek the support, it was on their terms, and at a time that they had determined was right to enter into their own recovery.

Partners and family (particularly mothers) were described as being the main supports throughout recovery. For example, one participant commented:

Yeah, I remember we'd go up to Mum's. She was a big support. And I'd go there when I initially was coming off, for days and just kind of eat and sleep and eat and sleep. Yeah, probably for a while actually. Like the beginning part, I can't even really remember to be honest. Yeah. And then slowly, my levels started to come right and yeah.. functioning a bit more normal. (Participant F, female)

Having someone who understood the recovery journey they were on and could be there for them through that time provided comfort and support for these participants.

Previous friends did not typically form part of the support, as they had become distanced through the period of MA use due to the user instead immersing themselves into the user community. Nor did former associates from the user community provide support when abstaining and recovering from MA addiction, as the user had needed to cut ties with them in support of their own recovery. Beside family and partners, the other people who provided support, through appropriate treatment services, were health practitioners.

\section{Getting away}

All participants said that the main thing required in getting away from MA

dependence was to remove themselves completely from the places, people and 
associates with whom they had become involved while using MA. The majority of participants stated that when they were using MA, they only associated with other MA users and became immersed in the user community. They would often push away family or friends who tried to reach out, or would isolate themselves and barely see those people due to wanting to remain hidden from being identified as a user by their loved ones. The longer the use, the more the dependence grew and the more they became immersed in the user community with others who were also using. As this woman explained: "I had to cut people off, you can't do it otherwise" (Participant A, female).

Emphasis was placed on the need to alter technology and social media so that people from the user community could not find them. For some this meant getting a new phone or deleting contacts and ignoring phone calls. Some deleted Facebook or made sure their profile information and security settings were highly anonymous. Two participants describe the lengths that were required to get away from old connections:

I changed my phone number and I also have a very private Facebook page with a different name so people couldn't find me. I have kept it low key and cut my ties. (Participant B, male)

Yeah, staying away from people that I used to know and be friends with. So, I don't answer any calls from any old people that I know. Um deleted all the numbers. And just, just got to refrain, try and stay away from the place where I was using a lot of meth in the area I lived, so I stay well clear of that place. Too many familiar faces. And yeah, not a good place to be for me. (Participant $C$, male)

Another main finding was the need to remove themselves from the location they had been living in and the places in which they had been using and dealing in MA. For some of the participants this meant having to move location. This often meant moving to stay with friends or family in a different part of Aotearoa New Zealand, or even a different country, and effectively starting over. They saw this as the only way to remove themselves completely from the situation and the triggers. For example:

We just had to move ourselves away from friends, places, I mean still now we go to town and there are places we remember stuff. So yeah just fresh beginning, just cutting ties with everything and friends and any associations to that lifestyle. (Participant F, female)

\section{Treatment}

Treatment was helpful to some participants, but only specific types of treatments were identified as having made a difference for them. The types of treatment that some of the participants sought included inpatient treatment, outpatient treatment and speaking to their general practitioner.

Two participants entered into inpatient (residential) drug and alcohol rehabilitation treatment centres, after not having success with outpatient treatment. Both completed their programmes but lapsed after getting out. They returned to the rehab programmes and achieved abstinence after this. The participants described inpatient treatment as a positive experience for them, one they found to be very helpful:

I found rehab was really good. That helped heaps. I was a live-in participant for four weeks the first time. And we were educated on side effects of drugs, and what damage can be done to your body and ways of coping before we left. (Participant C, male)

I just went in there full steam, you know, like I was there to do whatever it took to change my life, you know, and I did when I was in there, for five months. (Participant G, male) 
A majority of the participants had outpatient treatment (counselling treatment within the community) but participants described feeling that outpatient treatment had not helped them enough, and they did not feel they had been offered treatment that was specific to their issue with MA dependence, or that had enabled them to enter recovery. As one participant explained:

They mean well but their stuff's crap. No one's going to come out of the outpatient treatment that's offered with a new life. You know what I mean? Yeah. (Participant G, male)

Some participants spoke to their general practitioners (GP) about low moods and bouts of depression (which can be a cause of long-term MA use). The GP was generally unaware of the MA use and would prescribe medication for these participants. It seems the medication taken alongside MA was not replacing the need for MA or fulfilling its intended purpose, so the participants did not talk about having success with that approach. One participant who was open with the GP about his MA use, was given a diagnosis and placed on medication accordingly, and was able to effectively transition onto the prescribed medication and off the MA.

The participants who did not seek or receive any treatment all spoke about other factors that enabled them to cut down or go cold turkey from the drug, which are covered under the following theme of Personal Sources of Strength as well as factors that were received from the above-mentioned themes.

\section{Personal sources of strength}

Participants spoke about personal sources of strength that they had utilised in enabling their recovery journey. These included motivation and strength, fitness, spirituality, and smoking marijuana. All but one participant expressed the pivotal role that motivation and strength play in recovery.
This personal attribute is something that is seen as required to be able to abstain from MA, and enter into recovery from dependence on it. The dependence on the drug and loss of control had become clear for some of the participants, so when they decided there was a need to abstain from MA, they expressed that this would not have worked until they made a firm decision, and utilised motivation and strength in order to stick to that decision and see it through. As this woman explained:

I've just been strong in saying no and wanting my friends to respect my wishes. And just sticking to that "no means no", don't push me. Don't tell me to do something I just said I don't want to do, yeah. (Participant E, female)

For those who did not have treatment, the mental strength and motivation was a crucial factor in successfully entering, and being in, recovery. As explained by one of the participants:

Nah, I'm kind of stubborn like that. I just went cold turkey, eh. Cos I was still very, it's hard to explain, but very opinionated, and almost arrogant. Like I thought, you know, even though I wasn't high, it was still in my system and I was still really, you know it makes you very full of yourself. You know, you think you're untouchable and that. It's kind of like, I made the decision to do it, so I could make the decision to put it down. (Participant B, male)

Some of the participants explained that physical activity helped distract them from using MA and helped them to feel better mentally and physically. This also linked in with getting outdoors, being in nature and feeling more physically alive and able. They said this helped them to feel healthier and have more hope for their future while their recovery journey progressed:

Definitely, like keeping active and stuff and going to the gym. I didn't really 
go to the gym too much near the end, because I put on a lot of weight obviously afterward, from being on it and then being depressed and then just sleeping and then just being quite reserved and stuff. Yeah, but still getting out, like we bought bikes and just getting out there and getting active and walking the dog. (Participant F, female)

Fitness did help a lot. You know, with anxiety and stuff like that. Meth, you know. Later on, in life, I learnt what fitness was. I never had done any fitness. (Participant D, male)

For some participants, spirituality was a part of their recovery journey, one in which they established a connection with oneself or a higher power. One participant expressed the holistic wellbeing and centredness she tried to embrace for her sense of spirituality within, whereas another participant experienced spiritual enlightenment when finding God and Christianity. For those participants, spirituality was a part of their recovery in differing but equally important ways:

Well, I am quite a generally quite healthy person, so I am into like meditation, naturalness, going out in the bush ya know just really chilling and getting nice quality time. (Participant E, female)

And I was just going over the past. The past couple of years, that were probably the worst. And, and. And I remember making a bed and I remember actually saying a prayer that night. And I remember saying, "God, I've had enough. I need you to step in. I've had enough." (Participant G, male)

To abstain from MA, and cope with the withdrawal symptoms and psychological cravings for MA, some of the participants said they smoked marijuana as a personal prop. They explained that this could be seen as trading one drug for another but emphasised that it did help them. As explained:

I found smoking a lot of weed helped [to abstain from MA]. I've heard that about other people as well. When they come off, that weed really helps. I mean, maybe replacing one for the other but it does sort of help with the rigidness, the anxiety, when you have the jitters and can't sit still and feel agitated. Have a few puffs and it helps with that. (Participant $\mathrm{B}$, male)

Some participants also smoked cigarettes heavily in the initial stages of abstaining from MA but did not say that cigarettes helped with their withdrawal symptoms in the same way as marijuana did. The majority of participants who went cold turkey without treatment emphasised the role marijuana had in helping them with their recovery, over and above participants who were in treatment.

\section{Summary of themes}

There were disparate ways of coping described by the participants and it was discovered that recovery is multidimensional. Whilst the participants were on MA, they experienced negative consequences; entering recovery required them to try to understand the reasons behind their MA misuse such as escape from their personal issues, or enjoyment of the effects of the drug. Often drastic measures were needed in order for people to get away from MA use, such as moving location entirely and cutting ties with all former associates. The participants were at varying stages of their recovery processes, all identifying as being in recovery and having abstained for six months and more.

\section{Discussion}

Many theories and models exist to explain the phenomenon of drug dependence, but it does remain a subjective human experience. There is a shortage of qualitative 
research aimed at understanding the lived consumer perspective of recovery from MA dependence but the findings of this study are consistent with the multiple overseas studies that suggest that abstinence is an important aspect of recovery, but not sufficient (Bloom, 2016; Costello et al., 2018; Halkitis, 2009; Shinebourne \& Smith, 2010). In this study, four significant themes described the general experiences of the participants: Support, Getting Away, Treatment, and Personal Sources of Strength.

Families were the main source of support for these participants as they recovered from their MA use. Despite the participants having become distanced from all previous social connections whilst they were on MA and immersed in the user community, family members, particularly mothers, provided essential support during recovery. Treatment initiatives could benefit from helping individuals reconcile with families and allowing access to support networks, to encourage successful recovery. Individuals who do not have close support could be at more risk of not successfully abstaining from MA or relapsing and returning to the user community. Further research would be useful to enquire into the close support needs of those who do not have supportive family members.

All participants emphasised that, to abstain from MA and enter recovery, it was essential to get away from the user community and the associations made through it. The companionship gained through the user community meant it was not easy for some of the participants to no longer associate with those people. In the case that individuals are struggling to get away from MA dependence within their community or region, overseas studies suggest that residential rehabilitation centres would be beneficial—especially for MA users who have unstable or no accommodation, poor or absent social supports, co-existing mental health problems or have had failed attempts in the community (McKetin et al., 2018).
Treatment was a theme that was discussed with the participants, but only some found the treatment provided was helpful. More specifically, it seemed that inpatient treatment was deemed helpful, but outpatient treatment was not. The two participants who entered in-patient treatment spoke highly of the support they received from the practitioners and treatment providers within the rehabilitation programmes they experienced. The support they received included emotional support, psychological support, and knowledge about addiction and recovery. There is a barrier in Aotearoa New Zealand because treatment for MA dependence is not easily available. There are a limited number of rehabilitation centres that provide treatment specifically for MA dependence and these have criteria to meet before being accepted for entry, with possibly waiting lists, and financial payment required.

Those who did not have any treatment relied more heavily on their own personal strengths, such as motivation to be successful. There was a determination in some of these individuals that meant they could abstain from MA, without treatment, but they created a sense of being in the world that was conducive to getting away from their lifestyle, whilst finding other approaches that helped them. Drawing on their own resources gave participants a sense of personal self-worth and confidence that has been found to support recovery in previous studies (Bloom, 2016; Costello et al., 2018). This sense of personal strength may be an important component of ongoing recovery.

\section{Limitations}

The methodology allowed the first author to gain knowledge on the participants' lived experience of the phenomenon of drug dependence recovery and interpret their lived experience into findings. The quality of the data gathered in qualitative research is highly subjective. Having individual perspectives and interpretations can lead 
to detailed findings but these may also be interpreted in different ways. As there was just one researcher involved with the data collection and analysis in this study, additional research is required to validate the findings.

Overseas studies have also found aspects including daily structure, increased selfworth and confidence, employment, and life satisfaction as areas that were part of recovery (Costello et al., 2018; Radcliffe \& Tompkins, 2016). These aspects were not among the main findings of our study. This may be due to the small sample size; further research with a wider range of participants will contribute to our understandings of recovery needs.

The small sample size may not reflect important differences between ethnic groups, age groups, or social groups and this limitation also calls for further research to develop these understandings. Because there is a shortage of research on MA treatment and recovery within Aotearoa New Zealand, further research conducted within the country would be beneficial.

\section{Conclusions}

This research provides first-hand insight into the approaches, strategies, and treatment that the seven participants deemed helpful for their recovery. The lack of knowledge on appropriate treatment and recovery for MA in Aotearoa New Zealand is a concern, and this research should provide a valuable base of information on which further research can be conducted.

\section{Accepted 31 May 2021}

Published 1 September 2021

\section{References}

Alcohol Drug Helpline NZ. (2019). Helpline services. https:// alcoholdrughelp.org.nz/helpline/

Bloom, K. (2016). Turning points: The lived experience of addiction recovery [Master's thesis]. Clark University. https://commons.clarku.edu/idce_masters_papers/137/
Cannon, E. (2018). How to address methamphetamine abuse in the United States: Nurses leading comprehensive care. MEDSURG Nursing, 27(2), 103-107.

Cobzaru, L. (2010). Effective interventions for methamphetamine users [Unpublished doctoral dissertation]. Massey University.

Costello, M., Sousa, S., Ropp, C., \& Rush, B. (2020). How to measure addiction recovery? Incorporating perspectives of individuals with lived experience. International Journal of Mental Health and Addiction, 18(9), 599-612.

Drug Abuse. (2019). Understanding tolerance, dependence, and addiction. https://drugabuse.com/addiction/

Dual Recovery Network. (2016). Educational provider for methamphetamine dependence. https://www.drn.org.nz/ index.html

European Monitoring Centre for Drugs and Drug Addiction. (2018). Methamphetamine users in Europe. http:// www.emcdda.europa.eu/topics/pods/responses-formethamphetamine-users

Fields, R. (2017). Drugs in perspective (9th ed). McGraw-Hill Education.

Flick, U. (2014). The SAGE handbook of qualitative data analysis. SAGE Publications.

Gordon, G. D., \& De Jong, G. (2018). Gaps in the ice: Methamphetamine in Australia; its history, treatment, and ramifications for users and their families. Journal of Mental Health Nursing, 27(6), 1861-1868.

Halkitis, P. (2009). Methamphetamine addiction. American Psychological Association.

Jenner, L., \& Lee, N. (2008). Treatment approaches for users of methamphetamine: A practical guide for frontline workers. Australian Government Department of Health and Ageing.

King, J. (2014). Review of outcomes for clients who use methamphetamine at Higher Ground Rehabilitation Centre. http://www.higherground.org.nz/wp-content/ uploads/2014/09/Review-of-Outcomes-for-clients-whouse-Methamphetamine.pdf

Māori and Addiction Treatment Services. (2012). Final draft report. https://www.matuaraki.org.nz/uploads/files/ resource-assets/hui-final-draft-report-to-NCAT.pdf

McKetin, R., Kothe, A., Baker, L. A., Lee, N. K., Ross, J., \& Lubman, L. D. (2018). Predicting abstinence from methamphetamine use after residential rehab: Findings from the meth treatment evaluation study. Drug and Alcohol Review, 37(1), 70-78.

Meth Xpert NZ. (2019). Meth and drug statistics. https://www. methxpert.co.nz/new-zealand-drug-and-meth-statistics. html

Miller, G. P., Strang, J., \& Miller, M. P. (2010). Addiction research methods. Blackwell Publishing.

Moustakas, C. (1994). Phenomenological research methods. Sage Publications.

New Zealand Drug Foundation. (2018). Methamphetamine in NZ. https://www.drugfoundation.org.nz/policy-andadvocacy/meth-in-nz/

New Zealand Herald. (2019). Fighting the demon: Inside New Zealand's meth crisis. https://www.nzherald. co.nz/indepth/national/new-zealands-fight-againstmethamphetamine/ 
New Zealand Ministry of Health. (2010). Matua Raki 2010: Interventions and treatment for problematic use of methamphetamine and other amphetamine-type stimulants. Ministry of Health.

New Zealand Ministry of Health. (2013). Matua Raki 2013: Interventions and treatment for problematic use of methamphetamine and other amphetamine-type stimulants. Ministry of Health.

Radcliffe, P., \& Tompkins, C. (2016). "Once you've been there, you're always recovering": Exploring experiences, outcomes, and benefits of substance misuse recovery. Drugs and Alcohol Today Journal, 16(1), 29-38.

Red Door Recovery. (2018). Residential Treatment Centre. http://reddoorrecovery.co.nz

Roll, J., Rawson, R., Ling, W., \& Shoptaw, S. (2009). Methamphetamine addiction. The Guilford Press.

Savin-Baden, M., \& Major, H. C. (2013). Qualitative research. Routledge.

Shinebourne, P., \& Smith, J. (2010). "It is just habitual": An interpretative phenomenological analysis of the experience of long-term recovery from addiction. International Journal of Mental Health and Addiction, 9(3), 282-295.

Smith, J. A. (2008). Qualitative psychology (2nd ed.). Sage Publications.

Stoneberg, D., Shukla, R., \& Magness, M. (2017). Global methamphetamine trends: An evolving problem. International Criminal Justice Review, 28(2), 136-161.

UNODC. (2004). World Drug Report 2004. https://www. unodc.org/pdf/WDR_2004/WDR_2004_presentation.pdf

Weisheit, R., \& William, W. (2009). Methamphetamine: History, pharmacology, and treatment. Hazelden. 